\title{
Safety and efficacy of multiple site thoracic paravertebral nerve block vs. modified pectoral nerve block for postoperative analgesia after modified radical mastectomies-a randomised controlled trial
}

\author{
Viji S. Pillai ${ }^{1, *}$, Bhagyalakshmi Ramesh², Silvy Anna Varughese ${ }^{3}$ \\ ${ }^{\mathbf{1}}$ Associate Professor, ${ }^{2}$ Assistant Professor, ${ }^{3}$ Senior Resident, Dept. of Anaesthesiology, Regional Cancer Centre,
} Thiruvananthapuram, Kerala, India

*Corresponding Author:

Email: pillaiviji6@gmail.com

Received: $12^{\text {th }}$ March, 2018

Accepted: $1^{\text {st }}$ May, 2018

\begin{abstract}
This study compares the safety and efficacy of multiple level thoracic paravertebral block and modified Pecs block for postoperative pain relief after Modified Radical Mastectomies.

After Institutional Review Board [IRB] and Human Ethics Committee [HEC approval, the study was conducted on 41 patients who were scheduled to undergo modified radical mastectomy. Written informed consent was taken. Female patients in the age group of 18-75 years who were ASA grade I and II were randomly allocated to two groups to either receive Ultrasound guided Thoracic paravertebral block TPVB at T2 and T4 levels (Group 2) or an ultrasound guided modified PECS block (Group 1) preoperatively in the block room.

Paravertebral block was given at two vertebral levels T2 $(10 \mathrm{ml})$ and T4 level $(10 \mathrm{ml})$ under ultrasound guidance utilising the parasagital in-plane technique. Modified PEC block was administered to patients in the PEC group.10 ml of $0.5 \%$ Ropivacaine for Pec1 and 20ml for Pec 2. In all patients surgery was done under GA. Pain was assessed with Numerical Rating Scale (NRS) on a 0 to 10 scale, for 24 hours after surgery, at $0,0.5,1,1.5,2,4,6,12$ and 24 hours after surgery by a blinded observer.

Rescue opioid and Paracetamol requirement was recorded. The patients were also monitored for any specific block related complications.

Primary outcome measures were the numerical rating scores for pain and rescue analgesic requirement. Secondary outcome measures was looking for any block related complications. Demographic categorical variables were expressed in frequency and percentage, and the continuous variables were reported in terms of mean and standard deviation. The comparison of pain scores between the thoracic paravertebral nerve block and modified pectoral nerve block was done using Mann-Whitney U test. Mean duration of analgesia and mean 24 hour Morphine, Paracetamol consumption was compared using Student's $t$ test.

There was no statistically significant difference between the two groups with respect to the pain scores at $0,0.5,1,1.5,2,4,6,12$, and 24 hours at rest or with movement of the ipsilateral arm. The 24 hour Morphine consumption was not also statistically significant. [pec gp,mean 2.17, SD 1.09, tpvb gp mean 2 SD 0.77, p value 0.752.] Paracetamol consumption in the initial 24 hours after surgery was also not statistically significant between the two groups (pec gp. $36.8 \%$ and tpvb gp. 50\% patients received paracetamol with a $\mathrm{p}$ value of 0.523 ). We found both the blocks, modified Pecs and multiple level paravertebral block were equally effective in treating postoperative pain.
\end{abstract}

Keywords: Modified pectoral nerve block, Multiple level thoracic paravertebral block, Postoperative analgesia, Modified radical mastectomy.

\section{Introduction}

The age adjusted rate and mortality of breast cancer among Indian females is 25.8 and 12.7 per 100,000 women respectively. ${ }^{11}$ Modified radical mastectomy, can cause considerable pain in the immediate postoperative period and also has the potential to cause chronic pain ${ }^{1}$. Adequate pain control in the acute postoperative period is of paramount importance for patients' wellbeing, shorter hospital stay and also to prevent chronic pain due to the fact that inadequately treated postoperative pain is an important predictor of chronic pain., ${ }^{2,3}$ Many regional anaesthesia options are now available for the anaesthesiologist to choose from for breast surgeries. A proper understanding of the nerve supply and analgesic requirements for various breast surgical procedures is necessary to choose the correct regional technique. In our institution, a tertiary care cancer hospital in South India an average of six breast surgeries are done daily of which 3 to 4 will be modified radical mastectomies. These patients are offered General Anaesthesia with a peripheral nerve block, either paravertebral block or PEC block or local infiltration.

Thoracic paravertebral block (TPVB) has been well researched and has been found to provide not only excellent analgesia in breast surgeries but also has less severe chronic pain and thereby good physical and mental health related quality of life after surgery. ${ }^{4-7}$ Pectoral nerve block is an ultrasound guided interfascial plane block as described by Blanco in 2011 for postoperative pain relief in breast surgeries..$^{8-10}$ Recently a few studies have compared the safety and efficacy of the two blocks. It was observed that modified Pecs block provides superior postoperative analgesia than 
TPVB in radical mastectomies with axillary clearance. (Wahba and Kamal 2013; Kulhari S et al 2016). Our study compares the safety and efficacy of multiple level thoracic paravertebral block and modified Pecs block for postoperative pain relief after Modified Radical Mastectomies.

\section{Materials and Methods}

After Institutional Review Board (IRB.No.06/2017/03) and Human Ethics committee (HEC No.14/2017) approval the trial was registered at clinical trials registry of India (CTRI/2018/02/011757 Principal investigator: Dr. Viji S. Pillai Date of registration: 07/02/2018.).The study was conducted on 41 patients who were scheduled to undergo modified radical mastectomy after obtaining written informed consent. Female patients in the age group of 18-75 years who were ASA grade I and II were randomly allocated to two groups to either receive or an ultrasound guided modified PECS block (Group 1) or Ultrasound guided multiple level Thoracic paravertebral block at T2 and T4 levels (Group 2) preoperatively in the block room. Patients were randomised into two groups with computer generated random numbers in sealed opaque envelopes which were opened before administering the blocks. Patients with preexisting local site infection, coagulopathy, morbid obesity (BMI>35), allergy to local anaesthetic were excluded from the study. Patients with other major organ system dysfunction were also excluded from the study. Patients were reviewed in the preoperative day in the ward by one of the investigators. Procedure and Numerical Rating Score is then explained to the patient and informed consent is obtained. All the patients were kept nil per oral for 6 hours before procedure. They received Alprazolam $0.25-0.5 \mathrm{mg}$ on the night before and Pantoprazole 40mg on the morning of surgery. Prior to the block patient was monitored using standard ECG, SpO2 and NIBP measurements. After securing an intravenous line they received premedication with $1 \mathrm{mg}$ of Midazolam and Fentanyl 30 microgram.

Modified PEC block was administered to patients in the group 1. Under strict aseptic precautions, patient in supine position the probe is placed below the lateral part of the clavicle, similar to the probe position for infraclavicular brachial plexus block and then moving inferolaterally. After recognition of the appropriate anatomical structures, the skin puncture point is infiltrated with $2 \%$ lignocaine, then the block is performed by using a 22-gauge Quinke's needle. The needle is advanced to the tissue plane between the pectoralis major and pectoralis minor muscle at the vicinity of the pectoral branch of the acromiothoracic artery, and $10 \mathrm{~mL}$ of $0.5 \%$ ropivacaine deposited. In a similar manner, $20 \mathrm{~mL}$ is deposited at the level of the third rib between the pectoralis minor and Serratus Anterior muscle. Thoracic Paravertebral block with Ropivacaine $(0.5 \%)$ was given to patients in group 1 .
Procedure was done with patient in sitting position under strict aseptic precautions. Paravertebral block was given at two vertebral levels T2 $(10 \mathrm{ml})$ and T4 level $(10 \mathrm{ml})$ under ultrasound guidance utilising the parasagital in plane technique. Pleural displacement on injection of the drug is taken as confirmation for correct drug deposition.

After administering the blocks the patient's vitals, heart rate, blood pressure, $\mathrm{SpO} 2$ will be monitored continuously. Response to pinprick sensations in the thoracic dermatomes T1 - T6 at 20 minutes and 30 minutes after the block was noted.

Patients in both the groups underwent surgery with general anaesthesia standardized according to our centre's current clinical practice. General Anaesthesia was induced with Fentanyl 2 microgm $/ \mathrm{kg}$, Xylocard $1.5 \mathrm{mg} / \mathrm{kg} \mathrm{IV}$, Propofol (titrated to verbal response), Vecuronium $0.1 \mathrm{mg} / \mathrm{kg}$ for muscle relaxation and trachea intubated with appropriate sized ETT. Anaesthesia maintained with Sevoflorane and Oxygen, Nitrous oxide mixture with 1 MAC., FiO2 0.4 and ventilation titrated to maintain ETCO2 $35-40 \mathrm{mmHg}$, with low fresh gas flows. Fentanyl 0.5 microgram $/ \mathrm{kg}$ bolus will be given if the mean blood pressure or heart rate exceeded $20 \%$ of the baseline recordings, repeated once more if it does not return to baseline values after 10 minutes. After surgery patients were reversed with Neostigmine $2.5 \mathrm{mg}$ and Glycopyrrolate $0.4 \mathrm{mg}$ and extubated after good respiratory efforts and stable vitals. Patients were then shifted to the recovery room.

Post-operative pain was assessed in the recovery room and periodically after discharge to the ward until 24 hours postoperatively by another observer who was blinded regarding the block performed. Pain was assessed with Numerical Rating Scale (NRS) on a 0 to 10 scale, where 0 means no pain and 10stands for the maximum pain ever experienced for 24 hours after surgery, at $0,0.5,1,1.5,2,6,12$ and 24 hours after surgery.

Morphine $1.5 \mathrm{mg}$ bolus was given to treat postoperative pain. Paracetamol $1 \mathrm{gm}$ IV was given if pain did not subside after two boluses of Morphine. Rescue opioid and Paracetamol requirement was recorded. The patients were also monitored for any specific block related complications like local anaesthetic toxicity, pleural puncture, vascular puncture, pneumothorax and intrathecal spread of the drug.

\section{Statistical Methods}

Sample size was calculated with Master 2.0 sample size software. Sample size calculation was done based on the 24 hour postoperative Morphine consumption from a previous study by Kulhari et al.

Data was entered in Microsoft Excel sheet. Normality of data tested with P-P probability plot and histogram. Demographic categorical variables were expressed in frequency and percentage, and the continuous variables were reported in terms of mean 
and standard deviation. The comparison of pain scores between the thoracic paravertebral nerve block and modified pectoral nerve block was done using MannWhitney U test. Mean duration of analgesia and mean 24 hour Morphine, Paracetamol consumption was compared using Student's t test.

\section{Results}

Both groups were comparable with respect to age, body mass index, ASA status and the duration of surgery. The post block haemodynamic status remained stable throughout the period of monitoring. (Table 1)

There was no statistically significant difference between the two groups with respect to the pain scores at $0,0.5,1,1.5,2,4,6,12$, and 24 hours at rest or with movement of the ipsilateral arm. (Table 2, 3 ) The 24 hour Morphine consumption was not also statistically significant. [pec gp, mean 2.17, SD 1.09, tpvb gp mean 2 SD 0.77, p value 0.752.] Paracetamol consumption in the initial 24 hours after surgery was also not statistically significant between the two groups (pec gp. $36.8 \%$ and tpvb gp. 50\% patients received paracetamol with a $\mathrm{p}$ value of 0.523 ). (Table 4)

Time (in hours) to first analgesic was 5.667 (8.84) in the Pecs group and 2.985 (3.55) in the paravertebral group with a p value of 0.335 which was not significant. (Table 5)

There was a significant difference between two groups at the level of T2 dermatome block with $31.6 \%$ blocked in the PEC gp. and $80 \% \%$ in Tpvb with a $p$ value of 0.006 .

\section{Consort Diagram}

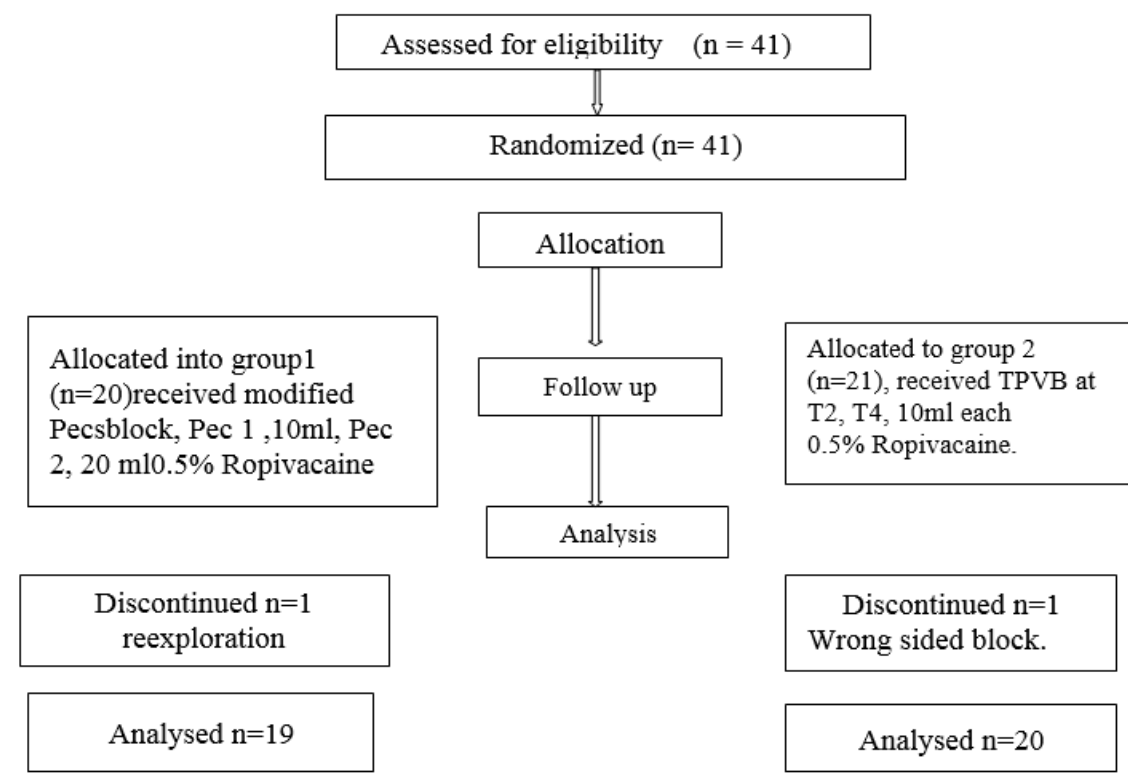

Table 1: Demographic variables comparison Student's t test

\begin{tabular}{|l|c|c|c|c|c|}
\hline Block & group & N & Mean & Std. Deviation & P-value \\
\hline \multirow{2}{*}{ Age } & 1 & 19 & 49.42 & 10.09 & \multirow{2}{*}{0.347} \\
\cline { 2 - 5 } & 2 & 20 & 52.40 & 9.46 & \\
\cline { 2 - 5 } BMI & 1 & 19 & 24.37 & 3.43 & 0.425 \\
\cline { 2 - 5 } & 2 & 20 & 25.19 & 2.92 & \\
\hline
\end{tabular}

Table 2: NRS mean values [Rest]

\begin{tabular}{|l|c|c|c|c|c|c|c|c|c|}
\hline Time & 0 & 0.5 & 1 & 1.5 & 2 & 4 & 6 & 12 & 24 \\
\hline PEC NRS & 0.58 & 1 & 1.11 & 1.42 & 1.42 & 1.89 & 1.89 & 1.68 & 1.68 \\
\hline TPVB NRS & 0.9 & 1.15 & 1.3 & 1.4 & 1.55 & 1.55 & 1.95 & 1.65 & 1.85 \\
\hline
\end{tabular}

Table 3: NRS mean values [Movement]

\begin{tabular}{|l|c|c|c|c|c|c|c|c|c|}
\hline Time & 0 & 0.5 & 1 & 1.5 & 2 & 4 & 6 & 12 & 24 \\
\hline PEC & 1 & 1.74 & 1.53 & 2.26 & 2.11 & 2.58 & 2.68 & 2.37 & 2.47 \\
\hline TPVB & 0.9 & 1.35 & 1.65 & 2.05 & 1.85 & 2 & 2.3 & 2.2 & 2.4 \\
\hline
\end{tabular}


Table 4: Paracetamol consumption in 24 hours

\begin{tabular}{|c|c|c|c|c|c|c|}
\hline \multirow{2}{*}{\multicolumn{3}{|c|}{ 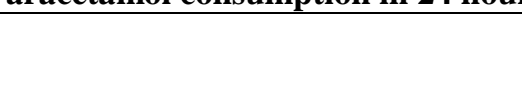 }} & \multicolumn{2}{|c|}{ PCM } & \multirow[t]{2}{*}{ Total } & \multirow[t]{2}{*}{ P-value } \\
\hline & & & Not given & Given & & \\
\hline \multirow[t]{4}{*}{ Block } & \multirow[t]{2}{*}{1} & Count & 12 & 7 & 19 & \\
\hline & & $\%$ within Block & $63.2 \%$ & $36.8 \%$ & $100.0 \%$ & \\
\hline & \multirow[t]{2}{*}{2} & Count & 10 & 10 & 20 & 0.523 \\
\hline & & $\%$ within Block & $50.0 \%$ & $50.0 \%$ & $100.0 \%$ & \\
\hline \multirow{2}{*}{\multicolumn{2}{|c|}{ Total }} & Count & 22 & 17 & 39 & \\
\hline & & $\%$ within Block & $56.4 \%$ & $43.6 \%$ & $100.0 \%$ & \\
\hline
\end{tabular}

Table 5: Student's t test for time to $1^{\text {st }}$ analgesic request, 24 hour morphine consumption

\begin{tabular}{|l|c|c|c|c|c|}
\hline \multicolumn{2}{|c|}{ Group } & N & Mean & Std. Deviation & P-value \\
\cline { 2 - 6 } $\begin{array}{l}\text { Time to first } \\
\text { analgesic_hrs }\end{array}$ & 1 & 12 & 5.667 & 8.84 & 0.335 \\
\cline { 1 - 5 } Mor_mg & 2 & 12 & 2.958 & 3.55 & \\
\cline { 2 - 5 } & 2 & 9 & 2.17 & 1.09 & 0.752 \\
\hline
\end{tabular}

Forty one patients were recruited into the study of which one patient in the Pecs group had reexploration and was later excluded from the study. One patient in the Tpvb group had wrong sided block who underwent surgery under general anaesthesia, postoperative pain managed with, Morphine and NSAIDS. Thus there were twenty patients in the paravertebral group and 19 in the PEC group.

\section{Discussion}

Axillary dissection and nerve injury may play a major role in causing chronic pain after breast cancer surgery. ${ }^{12-15}$ Nerve injury can cause central sensitization and trigger the development of chronic pain. Since nerve blocks are capable of preventing central sensitisation we feel the blockade of intercostobrachial nerve, the main nerve supplying the axilla plays a major role in decreasing the incidence of chronic pain after axillary dissection. ICBN is almost always handled by the surgeon and suffers varying degrees of damage..$^{38}$ Hence we designed our technique of paravertebral block at two levels T2 and T4 with $10 \mathrm{ml}$ of Ropivacaine at each level to assure adequate block of the intercostobrachial nerve.

The breast receives its innervation from the lateral and anterior cutaneous branches of the second to the sixth intercostal nerves and from the supraclavicular nerves. Axilla is mainly supplied by the intercostobrachial nerve which in turn communicates with other intercostal nerves $\mathrm{T} 1, \mathrm{~T} 3$ and $\mathrm{T} 4$, and also with nerves from brachial plexus. ${ }^{20-22}$ The pectoral muscle forms the anterior axillary wall which is supplied by the medial and the lateral pectoral nerves, serratus anterior muscle supplied by long thoracic nerve forms part of the medial wall and lattissimusdorsi supplied by thoracodorsal forms part of the posterior wall.

The fascia covering the pectoralis major muscle is removed during modified radical mastectomy. ${ }^{1,40}$ This contributes to the postoperative pain which can be alleviated by the pectoral nerve blocks. In reconstructive breast surgeries with subpectoral prosthesis, there can be considerable postoperative pain due to pectoralis muscle stretching, for which the Pecs 1 block was originally described. ${ }^{8,9,25}$ The pectoral muscles, serratus anterior and lattisimusdorsi are retracted during axillary dissection which can cause intraoperative pain. ${ }^{1}$ The long thoracic nerve and thoracodorsal nerve may suffer injury during axillary dissection which can cause motor weakness of the muscles supplied by them. ${ }^{40}$ The contribution of these nerves to postoperative pain after axillary dissection has yet to be understood. Sacrificing the intercostobrachial nerve may be required for oncological clearance during surgery which in turn can cause varying degrees of sensory loss in the axilla and medial side of arm. ${ }^{16,40}$

A few previous studies have evaluated the efficacy of PEC and TPVB for surgeries of breast including axillary dissection. Kulhari $\mathrm{S}^{11}$ et al in their study comparing PEC and paravertebral block found that the PEC block was superior to paravertebral block with respect to the duration of analgesia, 24 hour morphine consumption and postoperative pain scores in the initial two hours after surgery. Wahba et $\mathrm{al}^{12}$ in a similar study comparing these two blocks had less opioid consumption at 24 hours, longer time to first analgesic with the Pecs group. They had lower pain scores on movement at initial 12 hours and higher pain scores at 18 and 24 hours with Pecsblock. Another randomised double blind trial comparing Pecsblock, TPVB, and local infiltration by KarthikSyal et al had lower pain scores and longer duration of analgesia with the paravertebral block group. ${ }^{23}$ Here the blocks were given after surgery under GA before extubation.

It is quite interesting to note that in all these studies the paravertebral block was given at $\mathrm{T} 4$ level as a single site injection without a catheter. The authors point out this single site injection of thoracic paravertebral block as a drawback for adequate spread of the local 
anaesthetic to the higher dermatomal levels owing to the fact that spread of drug can be variable with this technique. Richardson et $\mathrm{al}^{30}$ in their study found that $1.5 \mathrm{mg} / \mathrm{kg}$ bupivacaine $0.5 \%$ produced sensory loss at the level of injection with a mean superior spread of 1.4 (range, 0-4) dermatomes and a mean inferior spread of 2.8 (range, 0-7) dermatomes. Thoracic paravertebral anesthesia does not appear to be gravity-dependent, but there is a tendency for preferential caudal spread of somatic and sympathetic blockade. ${ }^{26,31-34}$ Eason and Wyat ${ }^{27}$ found that at least four intercostal spaces could be covered by a single $15-\mathrm{ml}$ injection of $0.375 \%$ bupivacaine. Single site injection at the T4 level may not always anaesthetise the cephalad dermatomes, especially at the $\mathrm{T} 2$ level. This is more important when there is axillary dissection since it involves much of tissue handling and has major contribution to postoperative pain. The ICBN is a thick nerve compared to the other intercostals and needs to be blocked separately when axillary clearance is done.

Hetta $\mathrm{DF}^{35}$ et al, in their study on Pectoralisserratusinterfascial plane block vs thoracic paravertebral block for unilateral radical mastectomy with axillary evacuation concluded that Pectoralisserratus interfascial plane block was safe and easy to perform and decreased intensity of postmastectomy pain, but it was inferior to thoracic paravertebral block. Kasimahanti R. ${ }^{36}$ et al in their study on Ultrasoundguided single vs double-level thoracic paravertebral block for postoperative analgesia in total mastectomy with axillary clearance concludes that patients receiving double-level TPVB had significantly less 24-hour analgesic consumption in the postoperative period than those in the single-level TPVB group. They attribute this could be due to significantly greater number of segments blocked in the double-level TPVB group. This is similar to our study outcome.

The results of the study by Sopena-Zubiria $\mathrm{LA}^{37}$ et al showed that the combination of the two techniques was associated with a better post-operative analgesic control immediately after surgery: pain level assessed with VAS was significantly lower in the group treated with the combination of pectoral and thoracic paravertebral block 8 hours but not 24 hours after surgery. Combination of the two techniques reported a reduced need for intra-operative sedation.

We had a wrong sided block in one patient in the paravertebral block group who was excluded from the study. Surgery was done under general anaesthesia ad postoperative pain managed with Morphine and Paracetamol. Being a para median block extra precautions has to be exercised to prevent this.

We had similar experience as that of Bakshi et al, ${ }^{39}$ with surgeons having difficulty to use electrocautery during axillary dissection in the Modified Pecs group. This was due to the presence of local anaesthetic drug in the tissue planes and they had to use higher currents or resort to bipolar cautery.
Modified Pecs block has the advantage of simple and safe technique with ultrasound whereas paravertebral block is an advanced level block and demands more procedural expertise. Single level paravertebral blocks may not adequately cover all dermatomes always as the spread of local anaesthetic is variable. So in our study we compared paravertebral block at two levels with modified Pecs block and found both techniques had comparable outcomes with respect to numerical rating scales, time to first analgesic request and rescue analgesic consumption.

\section{Conclusion}

We found both the blocks, modified Pecs and multiple level paravertebral block equally effective in treating postoperative pain without any statistically significant difference between the two groups. Trials with large sample sizes may bring out differences. We feel that blocking the intercostobrachial nerve with a dedicated T2 level paravertebral block has an important role in perioperative analgesia in breast surgeries with axillary dissection since it is the major nerve supply to axilla and also because it is implicated in chronic pain.

\section{References}

1. Woodworth GE, Ivie RMJ, Nelson_SM, Walker CM, Maniker RB. Perioperative Breast Analgesia: A Qualitative Review of Anatomy and Regional Techniques. Reg Anesth Pain Med. 2017;42(5):609-631.

2. Fassoulaki A, Melemeni A, Staikou C, Triga A, Sarantopoulos C. Acute postoperative pain predicts chronic pain and long-term analgesic requirements after breast surgery for cancer. Acta Anaesthesiol Belg. 2008;59:241-248.

3. Bokhari FN, McMillan DE, McClement S, Daeninck PJ Pilot study of a survey to identify the prevalence of and risk factors for chronic neuropathic pain following breast cancer surgery. Oncol Nurs Forum. 2012;39:E141-E149.

4. Karmakar MK, Samy W, Li JW, Lee A, Chan WC, Chen PP, Ho AM. Thoracic paravertebral block and its effects on chronic pain and health-related quality of life after modified radical mastectomy. Reg Anesth Pain Med. 2014;39(4):289-98.

5. Wu J, Buggy D, Fleischmann E, Parra-Sanchez I, Treschan T, Kurz A, Mascha EJ, Sessler DI. Thoracic paravertebral regional anesthesia improves analgesia after breast cancer surgery: a randomized controlled multicentre clinical trial. Can J Anaesth. 2015;62(3):241-51.

6. Pérez-González O, Cuéllar-Guzmán LF, Soliz J, Cata JP. Impact of Regional Anesthesia on Recurrence, Metastasis, and Immune Response in Breast Cancer Surgery: A Systematic Review of the Literature. Reg Anesth Pain Med. 2017;42(6):751-756.

7. Klein SM, Bergh A, Steele SM, Georgiade GS, GreengrassRA.Thoracic paravertebral block for breast surgery. Anesth Analg. 2000;90:1402-5.

8. Blanco R. The 'pecs block': a novel technique for providing analgesia after breast surgery. Anaesthesia. 2011;66(9):847-8.

9. Blanco R, Fajardo M, Parras Maldonado T. Ultrasound description of Pecs II (modified Pecs I): a novel approach to breast surgery. Rev Esp Anestesiol Reanim. 2012;59(9):470-5. 
10. Chakraborty A, Khemka R, Datta T. Ultrasound-guided truncal blocks: A new frontier in regional anaesthesia. Indian J Anaesth. 2016;60:703-11.

11. Malvia, S., Bagadi, S. A., Dubey, U. S. and Saxena, S. Epidemiology of breast cancer in Indian women. AsiaPac J Clin Oncol. 2017;13:289-295.

12. Andersen KG, Duriaud HM, Jensen HE, Kroman N, Kehlet H. Predictive factors for the development of persistent pain after breast cancer surgery. Pain. 2015;156:2413-2422.

13. Wallace MS, Wallace AM, Lee J, Dobke MK. Pain after breast surgery: a survey of 282 women. Pain. 1996;66:195-205.

14. E. L. Poleshuck, J. Katz, C. H. Andrus. Risk factors for chronic pain following breast cancer surgery: a prospective study. Journal of Pain, 2006;7(9):626-634.

15. R. Gärtner, M. Jensen, J. Nielsen, M. Ewertz, N. Kroman, and $\mathrm{H}$. Kehlet. Prevalence of and factors associated with persistent pain following breast cancer surgery. JAMAJournal of the American Medical Association. 2009;302(18):1985-1992.

16. Torresan RZ, Cabello C, Conde DM, Brenelli HB. Impact of the preservation of the intercostobrachial nerve in axillary lymphadenectomy due to breast cancer. Breast $J$. 2003;9(5):389-92.

17. 17.Vadivelu N, Schreck M, Lopez J, Kodumudi G, Narayan D. Pain after mastectomy and breast reconstruction. Am Surg. 2008;74:285-296.

18. Sarhadi NS, Shaw Dunn J, Lee FD, Soutar DS. An anatomical study of the nerve supply of the breast, including the nipple and areola. Br J Plast Surg. 1996;49(3):156-64.

19. Jaspars JJ, Posma AN, van Immerseel AA, Gittenbergerde Groot AC. The cutaneous innervation of the female breast and nipple-areola complex: implications for surgery. Br J Plast Surg. 1997;50:249-259.

20. Davies F, Gladstone RJ, Stibbe EP. The anatomy of the intercostal nerves. J Anat. 1932;66:323-333.

21. Kulhari S, Bharti N, Bala I, Arora S, Singh G. Efficacy of pectoral nerve block versus thoracic paravertebral block for postoperative analgesia after radical mastectomy: A randomized controlled trial. Br J Anaesth. 2016;117:3826.

22. Wahba SS, Kamal SM. Thoracic paravertebral block versus pectoral nerve block for analgesia after breast surgery. Egypt J Anaesth. 2014;30:129-35.

23. Kartik Syal, Ankita Chandel. Comparison of the postoperative analgesic effect of paravertebral block, pectoral nerve block and local infiltration in patients undergoing modified radical mastectomy: A randomised double-blind trial. Indian. 2017;61(8):643-648.

24. Greengrass R. Is PECS superior to PVB? Methodological flaws with study ESRA Academy. Mar 7, 2017;170348.

25. Desroches J1, Grabs U, Grabs D. Selective ultrasound guided pectoral nerve targeting in breast augmentation: How to spare the brachial plexus cords? Clin Anat. 2013;26:49-55.

26. Cheema SP, Ilsley D, Richardson J, Sabanathan S: A thermographic study of paravertebral analgesia. Anaesthesia. 1995;50:118-21.

27. Eason MJ, Wyatt R: Paravertebral thoracic block-a reappraisal. Anaesthesia. 1979;34: 638-42.

28. A. Schnabel, S. U. Reichl, P. Kranke, E. M. PogatzkiZahn, and P. K. Zahn. Efficacy and safety of paravertebral blocks in breast surgery: a meta-analysis of randomized controlled trials. British Journal of Anaesthesia. 2010;105(6):842-852.

29. Terkawi_AS, Tsang $\underline{\text { S }}$, Sessler_DI, Terkawi_RS, Nunemaker MS, Durieux ME, Shilling A, Improving Analgesic Efficacy and Safety of Thoracic Paravertebral Block for Breast Surgery: A Mixed-Effects MetaAnalysis, Pain Physician, 2015;18(5):E757-80.

30. Richardson J, Jones J, Atkinson R: The effect of thoracic paravertebral blockade on intercostal somatosensory evoked potentials. Anesth Analg. 1998;87:373-6.

31. Saito T, Den S, Cheema SP. A single-injection, multisegmental paravertebral block-extension of somatosensory and sympathetic blockinvolunteers. Acta Anaesthesiol Scand. 2001;45:30-33.

32. Albokrinov AA, Fesenko UA. Spread of dye after single thoracolumbar paravertebral injection in infants. A cadaveric study. Eur J Anaesthesiol. 2014;31:305-309.

33. Marhofer D, Marhofer P, Kettner SC. Magnetic resonance imaging analysis of the spread of local anesthetic solution after ultrasound-guided lateral thoracic paravertebral blockade: a volunteer study. Anesthesiology. 2013;118:1106-1112.

34. Cowie B, McGlade D, Ivanusic J, Barrington MJ. Ultrasound-guided thoracic paravertebral blockade: A cadaveric study. Anesth Analg. 2010;110:1735-9.

35. Hetta DF, Rezk KM. Pectoralis-serratusinterfascial plane block vs thoracic paravertebral block for unilateral radical mastectomy with axillary evacuation. Randomized controlled trial. J ClinAnesth. 2016;34:91-7.

36. Bhatia N, Singh G Ultrasound-guided single- vs doublelevel thoracic paravertebral block for postoperative analgesia in total mastectomy with axillary clearance.Randomized controlled trial. J Clin Anesth. 2016;33:414-21.

37. Sopena-Zubiria LA, Fernández-Meré LA, Valdés Arias C, Muñoz González F, Sánchez Asheras J, Ibáñez Ernández C. Thoracic paravertebral block compared to thoracic paravertebral block plus pectoral nerve block in reconstructive breast surgery. Rev Esp Anestesiol Reanim. 2012;59:12-17.

38. Monique A. Steegers, Bas Wolters, Andrea W, Evers Luc Strobbe, Oliver H, Wilder-Smith. Effect of Axillary Lymph Node Dissection on Prevalence and Intensity of Chronic and Phantom Pain after Breast Cancer Surgery. The Journal of Pain. 2008;9(9):813-822.

39. Bakshi SG, Karan N, Parmar V. Pectoralis block for breast surgery: A surgical concern?. Indian J Anaesth. 2017;61:851-2.

40. S.D. Nathanson, K. Rosso, D. Chitale, M. Burke. Lymph Node Metastasis Editedby: Aamir Ahmad Introduction to Cancer Metastasis P. 235-261. ISBN: 978-0-12-804003-4

How to cite this article: Pillai VS, Ramesh B, Varughese SA. Safety and efficacy of multiple site thoracic paravertebral nerve block vs. modified pectoral nerve block for postoperative analgesia after modified radical mastectomies-a randomised controlled trial. Indian $\mathbf{J}$ Clin Anaesth. 2018;5(3):445-450. 